QUARTERLY OF APPLIED MATHEMATICS

VOLUME LXVIII, NUMBER 3

SEPTEMBER 2010, PAGES 459-468

S 0033-569X(2010)01197-0

Article electronically published on June 4, 2010

\title{
INITIAL BOUNDARY VALUE PROBLEM FOR SEMILINEAR HYPERBOLIC EQUATIONS AND PARABOLIC EQUATIONS WITH CRITICAL INITIAL DATA
}

\author{
BY \\ XU RUNZHANG \\ College of Science, Harbin Engineering University, 150001, People's Republic of China
}

\begin{abstract}
We study the initial boundary value problem of semilinear hyperbolic equations $u_{t t}-\Delta u=f(u)$ and semilinear parabolic equations $u_{t}-\Delta u=f(u)$ with critical initial data $E(0)=d$ (or $J\left(u_{0}\right)=d$ ), $I\left(u_{0}\right)<0$, and prove that there exist non-global solutions under classical conditions on $f$.
\end{abstract}

1. Introduction. It is well known that the semilinear hyperbolic equations and semilinear parabolic equations are the most important nonlinear evolution equations in the area of mathematical physics (see the previous works 11, 2, [1], 7], 13, [15]). The following are examples of important problems that are considered in this paper:

$$
\begin{aligned}
& u_{t t}-\Delta u=f(u), \quad x \in \Omega, t>0, \\
& u(x, 0)=u_{0}(x), \quad u_{t}(x, 0)=u_{1}(x), \quad x \in \Omega, \\
& u(x, t)=0, \quad x \in \partial \Omega, t \geq 0
\end{aligned}
$$

and

$$
\begin{aligned}
& u_{t}-\Delta u=f(u), \quad x \in \Omega, t>0, \\
& u(x, 0)=u_{0}(x), \quad x \in \Omega, \\
& u(x, t)=0, \quad x \in \partial \Omega, t \geq 0 .
\end{aligned}
$$

A powerful technique for treating the above problems is the so-called "potential well method", which was established by Payne and Sattinger [11. The technique for proving the global nonexistence of solutions of abstract problems that include (1.1) and (1.2) was developed first in [2]. In [1] a stronger result for (1.1) and (1.2) was established, namely pointwise blow-up in finite time. In [3] and [14], the case for which $E(0)>0$ was first considered. A "blow-up" (global nonexistence result) was proved in these two papers

Received November 18, 2008.

2000 Mathematics Subject Classification. Primary 35L05, 35K05.

Key words and phrases. Semilinear hyperbolic equations, semilinear parabolic equation, critical initial data, potential wells, global nonexistence.

E-mail address: xurunzh@yahoo.com.cn 
by relying on the condition that $\left(u_{0}, u_{1}\right) \geq 0$. The upper bound for $E(0)$ required was smaller than the depth of the potential well.

The potential well theory has ignited considerable interest among researchers to treat various parabolic and hyperbolic equations. Because it is not possible to cite all of the more than one hundred papers on the subject, we refer the reader to [4]-[6], $[8]-[10$, [16]- 22] and the references therein. In [11] the authors assumed that $f(u)$ satisfies the following conditions:

$$
(H) \begin{cases}(i) \quad f \in C^{1}, f(0)=f^{\prime}(0)=0 ; \\ (i i) \quad(a) f(u) \text { is monotone and convex for } u>0, \text { concave for } u<0 \text { or } \\ & (b) f(u) \text { is convex for }-\infty<u<\infty \\ (\text { iii }) \quad & \text { There exist } p \text { and } \gamma \text { satisfying } 2<p+1 \leq \gamma<\frac{n+2}{n-2} \\ & \text { such that }(p+1) F(u) \leq u f(u) \text { and }|u f(u)| \leq \gamma|F(u)| \\ & \text { where } F(u)=\int_{0}^{u} f(s) \mathrm{d} s .\end{cases}
$$

And Payne and Sattinger defined

$$
\begin{aligned}
& W=\left\{u \in H_{0}^{1}(\Omega) \mid I(u)>0, J(u)<d\right\} \cup\{0\}, \\
& V=\left\{u \in H_{0}^{1}(\Omega) \mid I(u)<0, J(u)<d\right\}
\end{aligned}
$$

where

$$
\begin{aligned}
& J(u)=\frac{1}{2}\|\nabla u\|^{2}-\int_{\Omega} F(u) \mathrm{d} x, \\
& I(u)=\|\nabla u\|^{2}-\int_{\Omega} u f(u) \mathrm{d} x, \\
& d=\inf _{u \in \mathcal{N}} J(u), \mathcal{N}=\left\{u \in H_{0}^{1}(\Omega) \mid I(u)=0,\|\nabla u\| \neq 0\right\}, \\
& E(t)=\frac{1}{2}\left\|u_{t}\right\|^{2}+\frac{1}{2}\|\nabla u\|^{2}-\int_{\Omega} F(u) \mathrm{d} x=\frac{1}{2}\left\|u_{t}\right\|^{2}+J(u) .
\end{aligned}
$$

In the above and the following discussions we denote $\|\cdot\|_{L^{p}(\Omega)}$ by $\|\cdot\|_{p},\|\cdot\|_{2}$ by $\|\cdot\|$ and $(u, v)=\int_{\Omega} u v \mathrm{~d} x$. In [11], by introducing the above sets $W$ and $V$, as well as other functionals, Payne and Sattinger gave a series of properties of $W$ and $V$. Then, by using $V$, they proved the global nonexistence of solution for problem (1.1) and (1.2) in the case $E(0)<d$ (or $J\left(u_{0}\right)<d$ for problem (1.2) $), I\left(u_{0}\right)<0$. In early 1977, in [12, Levine was informed by the authors of [11] that both proofs of Theorem 2.3 (about uniqueness) and Lemma 2.7 (in the case $f$ is convex) in [11] are incorrect. Recently, some other incorrect proofs regarding both invariance of the set $V$ and global non-existence (see Lemma 4.2, Theorem 4.3 and Theorem 6.3 in [11]) were pointed out and corrected in 21] by introducing a family of potential wells. In the correction (ii) on page 2667 of [21, the authors indicate that $M^{\prime}(t)$ could always be negative. Of course that is correct, but on page 294 of [11, near the top of the page, Payne and Sattinger rule out this possibility. The remark in 21] may leave the impression that they did not consider this possibility. 
In [21] they required the nonlinear term $f(u)$ to satisfy

$$
\left(H_{a}\right) \begin{cases}(i) & f \in C^{1}, f(0)=f^{\prime}(0)=0 ; \\ (i i) \quad & f(u) \text { is monotone and convex for } u>0, \text { concave for } u<0 ; \\ & \text { There exist a } p \text { and } \gamma \text { satisfying } 2<p+1 \leq \gamma<\frac{n+2}{n-2} \\ & \text { such that }(p+1) F(u) \leq u f(u) \text { and }|u f(u)| \leq \gamma|F(u)|, \\ & \text { where } F(u)=\int_{0}^{u} f(s) \mathrm{d} s,\end{cases}
$$

a hypothesis that will also be in force here. Moreover, in [21, some new results were achieved on the invariant sets, specifically global existence of solution for the critical initial data $E(0)=d$ (or $\left.J\left(u_{0}\right)=d\right), I\left(u_{0}\right) \geq 0$ for problems (1.1) and (1.2). But in 21. the authors did not consider all the cases related to the critical initial data. Let us explain this in detail. The main results obtained in [21] are the following theorems:

TheOREM 1.1. Let $f(u)$ satisfy $\left(\mathrm{H}_{a}\right), u_{0}(x) \in H_{0}^{1}(\Omega), u_{1}(x) \in L^{2}(\Omega)$. Assume that $E(0)<d$. Then when $I\left(u_{0}\right)>0$ or $\left\|\nabla u_{0}\right\|=0$, problem (1.1) admits a global weak solution $u(t) \in L^{\infty}\left(0, \infty ; H_{0}^{1}(\Omega)\right)$ with $u_{t}(t) \in L^{\infty}\left(0, \infty ; L^{2}(\Omega)\right)$ and $u(t) \in W$ for $0 \leq$ $t<\infty$; and when $I\left(u_{0}\right)<0$, the problem does not admit any global weak solution.

Theorem 1.2. Let $f(u)$ satisfy $\left(\mathrm{H}_{a}\right), u_{0}(x) \in H_{0}^{1}(\Omega), u_{1}(x) \in L^{2}(\Omega)$. Assume that $E(0)=d, I\left(u_{0}\right) \geq 0$. Then problem (1.1) admits a global weak solution $u(t) \in$ $L^{\infty}\left(0, \infty ; H_{0}^{1}(\Omega)\right)$ with $u_{t}(t) \in L^{\infty}\left(0, \infty ; L^{2}(\Omega)\right)$ and $u(t) \in \bar{W}=W \cup \partial W$ for $0 \leq t<\infty$.

TheOREM 1.3. Let $f(u)$ satisfy $\left(\mathrm{H}_{a}\right), u_{0}(x) \in H_{0}^{1}(\Omega)$. Assume that $J\left(u_{0}\right)<d$. Then when $I\left(u_{0}\right)>0$ or $\left\|\nabla u_{0}\right\|=0$, problem (1.2) admits a global weak solution $u(t) \in$ $L^{\infty}\left(0, \infty ; H_{0}^{1}(\Omega)\right)$ with $u_{t}(t) \in L^{2}\left(0, \infty ; L^{2}(\Omega)\right)$ and $u(t) \in W$ for $0 \leq t<\infty$; and when $I\left(u_{0}\right)<0$, the problem does not admit any global weak solution.

TheOrem 1.4. Let $f(u)$ satisfy $\left(\mathrm{H}_{a}\right), u_{0}(x) \in H_{0}^{1}(\Omega)$. Assume that $J\left(u_{0}\right)=d, I\left(u_{0}\right) \geq 0$. Then problem (1.2) admits a global weak solution $u(t) \in L^{\infty}\left(0, \infty ; H_{0}^{1}(\Omega)\right)$ with $u_{t}(t) \in$ $L^{2}\left(0, \infty ; L^{2}(\Omega)\right)$ and $u(t) \in \bar{W}$ for $0 \leq t<\infty$.

However for the global existence of solution for either problem (1.1) with $E(0)=d$, $I\left(u_{0}\right)<0$ or problem (1.2) with $J\left(u_{0}\right)=d, I\left(u_{0}\right)<0$, there are no results in [21], [11] or any other literature. In [19] Enzo Vitillaro considered the more general case $\left\|u_{0}\right\|_{p}>\lambda_{1}$, $E(0) \leq E_{1}$ for a class of abstract evolution equations. But the critical case $E(0)=E_{1}$ depends on the presence of the damping term. Hence these problems are still open. One of the reasons is that one cannot easily obtain the non-global existence of solution solely by the method used for $E(0)<d$ or $J\left(u_{0}\right)<d$. For instance, in order to prove the non-global existence of solution to problem (1.1) with critical case $E(0)=d, I\left(u_{0}\right)<0$, one must ensure the invariance of set $V^{\prime}=\left\{u \in H_{0}^{1}(\Omega) \mid I\left(u_{0}\right)<0\right\}$ under the flow of (1.1) in the case of $E(0)=d$. It is natural to think whether we can use the method for case $E(0)<d$ to solve the problem with critical case $E(0)=d$. In the process of treating the case $E(0)<d$, by a contradiction method, we can suppose the invariance of the set $V^{\prime}$ does not hold; then there exists a $t_{0} \in(0, T)$ such that $I\left(u\left(t_{0}\right)\right)=0$ and $I(u)<0$ for $0<t<t_{0}$, where $T$ is the existence time of $u$. Hence we have $\left\|\nabla u\left(t_{0}\right)\right\| \geq r(1)$ and 
$J\left(u\left(t_{0}\right)\right) \geq d$. Unfortunately, this result does not contradict

$$
\frac{1}{2}\left\|u_{t}\right\|^{2}+J(u)=E(t) \leq E(0)=d, \quad 0 \leq t<T .
$$

Therefore this situation yields some difficulties, so we have to work out some new ideas in order to solve this problem.

The main purpose of this paper is to resolve the open problems mentioned above by using the potential well method. We prove that if $f(u)$ satisfies $\left(\mathrm{H}_{a}\right), E(0)=d, I\left(u_{0}\right)<0$ and $\left(u_{0}, u_{1}\right) \geq 0$ for problem (1.1); or $J\left(u_{0}\right)=d, I\left(u_{0}\right)<0$ for problem (1.2), then either problem (1.1) or problem (1.2) does not admit any global weak solution. So, we seek to give positive answers to the unsolved problems existing in [21] and [11.

2. Definitions and preliminary lemmas. In this section we give some definitions and recall some preliminary lemmas.

For (1.1) and (1.2) we define $J(u), I(u), E(t), d, W$ and $V$ as above. In addition, we define the functional related to the Nehari flow as

$$
I_{\delta}(u)=\delta\|\nabla u\|^{2}-\int_{\Omega} u f(u) \mathrm{d} x, \delta>0,
$$

the depth of the family of potential wells as

$$
\begin{gathered}
d(\delta)=\inf _{u \in \mathcal{N}_{\delta}} J(u), \\
\mathcal{N}_{\delta}=\left\{u \in H_{0}^{1}(\Omega) \mid I_{\delta}(u)=0,\|\nabla u\| \neq 0\right\}, \delta>0 ;
\end{gathered}
$$

and the family of potential wells as

$$
\begin{aligned}
& W_{\delta}=\left\{u \in H_{0}^{1}(\Omega) \mid I_{\delta}(u)>0, J(u)<d(\delta)\right\} \cup\{0\}, \quad 0<\delta<b ; \\
& V_{\delta}=\left\{u \in H_{0}^{1}(\Omega) \mid I_{\delta}(u)<0, J(u)<d(\delta)\right\}, \quad 0<\delta<b .
\end{aligned}
$$

Then some lemmas can be recalled.

Lemma 2.1 (21, [11]). Let $f(u)$ satisfy $\left(H_{a}\right)$. Then

(i) $|F(u)| \leq A|u|^{\gamma}$ for some $A>0$ and $\forall u \in \mathbb{R}$.

(ii) $|u f(u)| \leq \gamma A|u|^{\gamma}, \forall u \in \mathbb{R}$.

Lemma 2.2 ([21]). Let $f(u)$ satisfy $\left(H_{a}\right)$. Assume that $u \in H_{0}^{1}(\Omega)$ and $I_{\delta}(u)<0$. Then $\|\nabla u\|>r(\delta)$; in particular, if $I(u)<0$, then $\|\nabla u\|>r(1)$, where

$$
r(\delta)=\left(\frac{\delta}{a C_{*}^{\gamma}}\right)^{\frac{1}{\gamma-2}}, \quad a=\sup _{u \in \mathbb{R}, u \neq 0} \frac{u f(u)}{|u|^{\gamma}}, \quad C_{*}=\sup _{u \in H_{0}^{1}(\Omega), u \neq 0} \frac{\|u\|_{\gamma}}{\|\nabla u\|} .
$$

Lemma 2.3 ([21]). Let $f(u)$ satisfy $\left(H_{a}\right)$. For $d(\delta)$ we have

(i) $d(\delta) \geq a(\delta) r^{2}(\delta)$ for $a(\delta)=\frac{1}{2}-\frac{\delta}{p+1}, 0<\delta<\frac{p+1}{2}$.

(iii) $\lim _{\delta \rightarrow 0} d(\delta)=0$ and there exists a constant $b$ satisfying $\frac{p+1}{2} \leq b \leq \frac{\gamma}{2}$ such that $d(b)=0$ and $d(\delta)>0$ for $0<\delta<b$.

(iii) $d(\delta)$ is increasing on $0<\delta \leq 1$, decreasing on $1 \leq \delta \leq b$ and takes the maximum $d=d(1)$ at $\delta=1$.

Throughout this paper we employ the notion of weak solution defined below. 
Definition 2.4. $u=u(x, t)$ is called a weak solution of problem (1.1) on $\Omega \times[0, T)$ if $u \in L^{\infty}\left(0, T ; H_{0}^{1}(\Omega)\right)$ with $u_{t} \in L^{\infty}\left(0, T ; L^{2}(\Omega)\right)$ satisfying

(i) $\left(u_{t}, v\right)+\int_{0}^{t}(\nabla u, \nabla v) \mathrm{d} \tau=\int_{0}^{t}(f(u), v) \mathrm{d} \tau+\left(u_{1}, v\right), \forall v \in H_{0}^{1}(\Omega), t \in(0, T)$.

(ii) $u(x, 0)=u_{0}(x)$ in $H_{0}^{1}(\Omega)$.

(iii) $E(t) \leq E(0), \quad 0 \leq t<T$.

Definition 2.5. $u=u(x, t)$ is called a weak solution of problem (1.2) on $\Omega \times[0, T)$ if $u \in L^{\infty}\left(0, T ; H_{0}^{1}(\Omega)\right)$ with $u_{t} \in L^{2}\left(0, T ; L^{2}(\Omega)\right)$ satisfying

(i) $\left(u_{t}, v\right)+(\nabla u, \nabla v)=(f(u), v), \quad \forall v \in H_{0}^{1}(\Omega), t \in(0, T)$.

(ii) $u(x, 0)=u_{0}(x)$ in $H_{0}^{1}(\Omega)$.

(iii) $\int_{0}^{t}\left\|u_{\tau}\right\|^{2} \mathrm{~d} \tau+J(u) \leq J\left(u_{0}\right), \quad 0 \leq t<T$.

There is a difference between problem (1.1) and problem (1.2). For problem (1.2) we have the following Lemma 2.6 to guarantee the invariance of the set $V_{\delta}$. Although this invariance is not for the critical data $J\left(u_{0}\right)=d$, it will be shown to be sufficient to derive the nonexistence of global solution of (1.2). But for problem (1.1) this method does not appear to work.

Lemma 2.6 ([21]). Let $f(u)$ satisfy $\left(H_{a}\right), u_{0}(x) \in H_{0}^{1}(\Omega)$. Assume that $0<e<d$, $\left(\delta_{1}, \delta_{2}\right)$ is the maximal interval including $\delta=1$ such that $d(\delta)>e$ for $\delta \in\left(\delta_{1}, \delta_{2}\right)$. Then all weak solutions of problem (1.2) with $0<J\left(u_{0}\right) \leq e$ belong to $V_{\delta}$ for $\delta \in\left(\delta_{1}, \delta_{2}\right)$, provided $I\left(u_{0}\right)<0$.

As mentioned above, we need to give the invariance of set $V_{\delta}$ for problem (1.1) as follows.

Lemma 2.7. Let $f(u)$ satisfy $\left(H_{a}\right), u_{0}(x) \in H_{0}^{1}(\Omega), u_{1}(x) \in L^{2}(\Omega)$. Assume that $E(0)=$ $d$ and $\left(u_{0}, u_{1}\right) \geq 0$. Then the following set

$$
V^{\prime}=\left\{u \in H_{0}^{1}(\Omega) \mid I(u)<0\right\}
$$

is invariant under the flow of (1.1).

Proof. Let $u(t)$ be any weak solution of problem (1.1) with $E(0)=d, I\left(u_{0}\right)<0$ and $\left(u_{0}, u_{1}\right) \geq 0, T$ being the existence time of $u(t)$. Let us prove $I(u)<0$ for $0<t<T$. If it is false, then there exists a $t_{0} \in(0, T)$ such that $I\left(u\left(t_{0}\right)\right)=0$ and $I(u)<0$ for $0 \leq t<t_{0}$. Hence we have $\|\nabla u\|>r(1)$ for $0 \leq t<t_{0}$ and $\left\|\nabla u\left(t_{0}\right)\right\| \geq r(1)>0$. By the definition of $d$ we get $J\left(u\left(t_{0}\right)\right) \geq d$. From this and

$$
\frac{1}{2}\left\|u_{t}\left(t_{0}\right)\right\|^{2}+J\left(u\left(t_{0}\right)\right) \leq E(0)=d,
$$

we get $J\left(u\left(t_{0}\right)\right)=d$ and $\left\|u_{t}\left(t_{0}\right)\right\|^{2}=0$. Let $M(t)=\|u\|^{2}$. Then we have

$$
\dot{M}=2\left(u, u_{t}\right)
$$

with

$$
\begin{gathered}
\dot{M}(0)=2\left(u_{0}, u_{1}\right) \geq 0, \\
\ddot{M}(t)=2\left\|u_{t}\right\|^{2}-2 I(u)>0,0 \leq t<t_{0} .
\end{gathered}
$$


Hence $\dot{M}(t)$ is strictly increasing with respect to $t \in\left[0, t_{0}\right]$. This together with $\dot{M}(0)=$ $2\left(u_{0}, u_{1}\right) \geq 0$ gives $\dot{M}\left(t_{0}\right)=2\left(u\left(t_{0}\right), u_{t}\left(t_{0}\right)\right)>0$. This contradicts $\left\|u_{t}\left(t_{0}\right)\right\|=0$. So this completes this proof.

3. Main results and proof. In this section we state the main results and prove them.

Theorem 3.1. Let $f(u)$ satisfy $\left(H_{a}\right), u_{0}(x) \in H_{0}^{1}(\Omega), u_{1}(x) \in L^{2}(\Omega)$. Assume that $E(0)=d, I\left(u_{0}\right)<0$ and $\left(u_{0}, u_{1}\right) \geq 0$. Then the existence time of a weak solution for problem (1.1) is finite.

Proof. Let $u(t)$ be any weak solution of problem (1.1) with $E(0)=d, I\left(u_{0}\right)<0$ and $\left(u_{0}, u_{1}\right) \geq 0, T$ being the existence time of $u(t)$. Let us prove $T<\infty$. Arguing by contradiction, let us assume $T=+\infty$. Again, let

$$
M(t)=\|u\|^{2} .
$$

Then we have

$$
\dot{M}=2\left(u, u_{t}\right)
$$

with

$$
\begin{gathered}
\dot{M}(0)=2\left(u_{0}, u_{1}\right) \geq 0, \\
\ddot{M}(t)=2\left\|u_{t}\right\|^{2}-2 I(u), 0 \leq t<\infty .
\end{gathered}
$$

From (2.4) and $(p+1) F(u) \leq u f(u)$ we arrive at

$$
\frac{1}{2}\left\|u_{t}\right\|^{2}+\frac{p-1}{2(p+1)}\|\nabla u\|^{2}+\frac{1}{p+1} I(u) \leq \frac{1}{2}\left\|u_{t}\right\|^{2}+J(u)=E(t) \leq E(0)=d .
$$

Hence we have

$$
\begin{gathered}
\ddot{M}(t) \geq(p+3)\left\|u_{t}\right\|^{2}+(p-1)\|\nabla u\|^{2}-2(p+1) d \\
\geq(p+3)\left\|u_{t}\right\|^{2}+(p-1) \lambda_{1} M(t)-2(p+1) d, 0 \leq t<\infty, \\
\lambda_{1}=\inf _{u \in H_{0}^{1}(\Omega), u \neq 0} \frac{\|\nabla u\|^{2}}{\|u\|^{2}} .
\end{gathered}
$$

Eq. (3.1) and Lemma 2.7 yield $\ddot{M}(t)>0$ for $0 \leq t<\infty$ and $\dot{M}(t)$ is strictly increasing for $0 \leq t<\infty$. Hence for any $t_{0}>0$ we have $\dot{M}(t) \geq \dot{M}\left(t_{0}\right)>0$ for $t \geq t_{0}$, and

$$
M(t) \geq \dot{M}\left(t_{0}\right)\left(t-t_{0}\right)+M\left(t_{0}\right) \geq \dot{M}\left(t_{0}\right)\left(t-t_{0}\right), t \geq t_{0} .
$$

Therefore for sufficiently large $t$, we have $(p-1) \lambda_{1} M(t)>2(p+1) d$ and

$$
\ddot{M}(t)>(p+3)\left\|u_{t}\right\|^{2} .
$$

Hence

$$
\begin{gathered}
M \ddot{M}-\frac{p+3}{4}(\dot{M})^{2} \geq(p+3)\left(\|u\|^{2}\left\|u_{t}\right\|^{2}-\left(u, u_{t}\right)^{2}\right) \geq 0, \\
\left(M^{-\alpha}\right)^{\prime \prime}=\frac{-\alpha}{M^{\alpha+2}}\left(M \ddot{M}-(\alpha+1)(\dot{M})^{2}\right) \leq 0, \quad \alpha=\frac{p-1}{4} .
\end{gathered}
$$

From (3.3) it follows that there exists a $T_{1}>0$ such that

$$
\lim _{t \rightarrow T_{1}} M^{-\alpha}(t)=0,
$$


and

$$
\lim _{t \rightarrow T_{1}} M(t)=+\infty
$$

which contradict $T=+\infty$. The proof is completed.

From Theorem 1.2 and Theorem 3.1 we can get a sharp condition for global existence of solution for problem (1.1) with $E(0)=d$ as follows:

Theorem 3.2. Let $f(u)$ satisfy $\left(H_{a}\right), u_{0}(x) \in H_{0}^{1}(\Omega), u_{1}(x) \in L^{2}(\Omega)$. Assume that $E(0)=d,\left(u_{0}, u_{1}\right) \geq 0$. Then when $I\left(u_{0}\right) \geq 0$, problem (1.1) admits a global weak solution $u(t) \in L^{\infty}\left(0, \infty ; H_{0}^{1}(\Omega)\right)$ with $u_{t}(t) \in L^{\infty}\left(0, \infty ; L^{2}(\Omega)\right)$ and $u(t) \in \bar{W}=W \cup \partial W$ for $0 \leq t<\infty$; and when $I\left(u_{0}\right)<0$, the problem does not admit any global weak solution.

Theorem 3.3. Let $f(u)$ satisfy $\left(H_{a}\right), u_{0}(x) \in H_{0}^{1}(\Omega)$. Assume that $J\left(u_{0}\right)=d, I\left(u_{0}\right)<0$. Then the existence time of weak solution for problem (1.2) is finite.

Proof. Let $u(t)$ be any weak solution of problem (1.2) with $J\left(u_{0}\right)=d, I\left(u_{0}\right)<0, T$ being the existence time of $u(t)$. Let us prove $T<\infty$. Arguing by contradiction, we suppose $T=+\infty$. Let

$$
M_{1}(t)=\int_{0}^{t}\|u\|^{2} \mathrm{~d} \tau
$$

Then

$$
\ddot{M}_{1}(t)=2\left(u_{t}, u\right)=-2 I(u), \quad 0 \leq t<\infty .
$$

From (2.5) and

$$
\int_{\Omega} u f(u) \mathrm{d} x \geq(p+1) \int_{\Omega} F(u) \mathrm{d} x
$$

we get

$$
\begin{aligned}
& \int_{0}^{t}\left\|u_{\tau}\right\|^{2} \mathrm{~d} \tau+\frac{p-1}{2(p+1)}\|\nabla u\|^{2}+\frac{1}{p+1} I(u) \\
\leq & \int_{0}^{t}\left\|u_{\tau}\right\|^{2} \mathrm{~d} \tau+J(u) \leq J\left(u_{0}\right)=d .
\end{aligned}
$$

From (3.4) and (3.5) we have

$$
\begin{aligned}
\ddot{M}_{1}(t) & \geq 2(p+1) \int_{0}^{t}\left\|u_{\tau}\right\|^{2} \mathrm{~d} \tau+(p-1)\|\nabla u\|^{2}-2(p+1) d \\
& \geq 2(p+1) \int_{0}^{t}\left\|u_{\tau}\right\|^{2} \mathrm{~d} \tau+(p-1) \lambda_{1} \dot{M}_{1}(t)-2(p+1) d .
\end{aligned}
$$

Note that

$$
\begin{aligned}
\left(\int_{0}^{t}\left(u_{\tau}, u\right) \mathrm{d} \tau\right)^{2} & =\left(\frac{1}{2} \int_{0}^{t} \frac{\mathrm{d}}{\mathrm{d} \tau}\|u\|^{2} \mathrm{~d} \tau\right)^{2} \\
& =\frac{1}{4}\left(\|u\|^{4}-2\left\|u_{0}\right\|^{2}\|u\|^{2}+\left\|u_{0}\right\|^{4}\right) \\
& =\frac{1}{4}\left(\dot{M}_{1}^{2}(t)-2\left\|u_{0}\right\|^{2} \dot{M}_{1}(t)+\left\|u_{0}\right\|^{4}\right) .
\end{aligned}
$$


Hence we have

$$
\begin{aligned}
M_{1} \ddot{M}_{1}-\frac{p+1}{2}\left(\dot{M}_{1}\right)^{2} \geq & 2(p+1)\left[\int_{0}^{t}\|u\|^{2} \mathrm{~d} \tau \int_{0}^{t}\left\|u_{\tau}\right\|^{2} \mathrm{~d} \tau-\left(\int_{0}^{t}\left(u, u_{\tau}\right) \mathrm{d} \tau\right)^{2}\right] \\
& +(p-1) \lambda_{1} M_{1}(t) \dot{M}_{1}(t)-(p+1)\left\|u_{0}\right\|^{2} \dot{M}_{1}(t) \\
& -2(p+1) d M_{1}(t)+\frac{p+1}{2}\left\|u_{0}\right\|^{4} .
\end{aligned}
$$

From this and the Schwartz inequality we get

$$
\begin{aligned}
& M_{1} \ddot{M}_{1}-\frac{p+1}{2}\left(\dot{M}_{1}\right)^{2} \\
\geq & (p-1) \lambda_{1} M_{1}(t) \dot{M}_{1}(t)-(p+1)\left\|u_{0}\right\|^{2} \dot{M}_{1}(t)-2(p+1) d M_{1}(t) \\
= & \left(\frac{1}{2}(p-1) \lambda_{1} M_{1}(t)-(p+1)\left\|u_{0}\right\|^{2}\right) \dot{M}_{1}(t) \\
& +\left(\frac{1}{2}(p-1) \lambda_{1} \dot{M}_{1}(t)-2(p+1) d\right) M_{1}(t) .
\end{aligned}
$$

On the other hand, from $J\left(u_{0}\right)=d>0, I\left(u_{0}\right)<0$ and the continuity of $J(u)$ and $I(u)$ with respect to $t$, it follows that there exists a sufficiently small $t_{1}>0$ such that $J\left(u\left(t_{1}\right)\right)>0$ and $I(u)<0$ for $0 \leq t \leq t_{1}$. Hence $\left(u_{t}, u\right)=-I(u)>0$ and $\left\|u_{t}\right\|>0$ for $0 \leq t \leq t_{1}$. From this and the continuity of $\int_{0}^{t}\left\|u_{\tau}\right\|^{2} \mathrm{~d} \tau$ it follows that we can choose a $t_{1}$ such that

$$
0<d_{1}=d-\int_{0}^{t_{1}}\left\|u_{t}\right\|^{2} \mathrm{~d} t<d
$$

And by (2.5) we have

$$
0<J\left(u\left(t_{1}\right)\right) \leq d-\int_{0}^{t_{1}}\left\|u_{t}\right\|^{2} \mathrm{~d} t=d_{1}<d
$$

Thus if in Lemma 2.6 we take $t=t_{1}$ as the initial time, then we have $u(t) \in V_{\delta}$ for $\delta \in\left(\delta_{1}, \delta_{2}\right), t_{1} \leq t<\infty$, where $\left(\delta_{1}, \delta_{2}\right)$ is the maximal interval including $\delta=1$ such that $d(\delta)>d_{1}$ for $\delta \in\left(\delta_{1}, \delta_{2}\right)$. Hence we have $I_{\delta}(u)<0$ and $\|\nabla u\|>r(\delta)$ for $\delta \in\left(1, \delta_{2}\right)$, $t_{1} \leq t<\infty$, and $I_{\delta_{2}}(u) \leq 0,\|\nabla u\| \geq r\left(\delta_{2}\right)$ for $t_{1} \leq t<\infty$. Thus from (3.4) we get

$$
\begin{gathered}
\ddot{M}_{1}(t)=-2 I(u)=2\left(\delta_{2}-1\right)\|\nabla u\|^{2}-2 I_{\delta_{2}}(u) \\
\geq 2\left(\delta_{2}-1\right)\|\nabla u\|^{2} \geq 2\left(\delta_{2}-1\right) r^{2}\left(\delta_{2}\right) \equiv C\left(\delta_{2}\right), \quad t_{1} \leq t<\infty, \\
\dot{M}_{1}(t) \geq C\left(\delta_{2}\right)\left(t-t_{1}\right)+\dot{M}_{1}\left(t_{1}\right) \geq C\left(\delta_{2}\right)\left(t-t_{1}\right), \quad t_{1} \leq t<\infty, \\
M_{1}(t) \geq \frac{1}{2} C\left(\delta_{2}\right)\left(t-t_{1}\right)^{2}+M_{1}\left(t_{1}\right)>\frac{1}{2} C\left(\delta_{2}\right)\left(t-t_{1}\right)^{2}, \quad t_{1} \leq t<\infty .
\end{gathered}
$$

From (3.9) and (3.10) it follows that for sufficiently large $t$ we have

$$
\frac{1}{2}(p-1) \lambda_{1} M_{1}(t)>(p+1)\left\|u_{0}\right\|^{2}
$$

and

$$
\frac{1}{2}(p-1) \lambda_{1} \dot{M}_{1}(t)>2(p+1) d
$$


Thus (3.7) yields

which gives

$$
M_{1}(t) \ddot{M}_{1}(t)-\frac{p+1}{2}\left(\dot{M}_{1}(t)\right)^{2}>0
$$

$$
\left(M_{1}^{-\alpha}\right)^{\prime \prime}=\frac{-\alpha}{M_{1}^{\alpha+2}}\left(M_{1} \ddot{M}_{1}-(\alpha+1)\left(\dot{M}_{1}\right)^{2}\right) \leq 0, \quad \alpha=\frac{p-1}{2} .
$$

From this it follows that there exists a $T_{1}>0$ such that

$$
\lim _{t \rightarrow T_{1}} M_{1}^{-\alpha}(t)=0
$$

and

$$
\lim _{t \rightarrow T_{1}} M_{1}(t)=+\infty
$$

which contradicts $T=+\infty$.

From Theorem 1.4 and Theorem 3.3 we can obtain a sharp condition for global existence of solution for problem (1.2) with $J\left(u_{0}\right)=d$ as follows.

Theorem 3.4. Let $f(u)$ satisfy $\left(H_{a}\right), u_{0}(x) \in H_{0}^{1}(\Omega)$. Assume that $J\left(u_{0}\right)=d$. Then when $I\left(u_{0}\right) \geq 0$, problem (1.2) admits a global weak solution $u(t) \in L^{\infty}\left(0, \infty ; H_{0}^{1}(\Omega)\right)$ with $u_{t}(t) \in L^{2}\left(0, \infty ; L^{2}(\Omega)\right)$ and $u(t) \in \bar{W}=W \cup \partial W$ for $0 \leq t<\infty$; and when $I\left(u_{0}\right)<0$, the problem does not admit any global weak solution.

Acknowledgements. This work was supported by National Natural Science Foundation of China (10871055); Natural Science Foundation of Heilongjiang Province (A200702; A200810); Science and Technology Foundation of Education Office of Heilongjiang Province (11541276); Foundational Science Foundation of Harbin Engineering University. I especially appreciate the referee's valuable suggestions and even guidance. The referee provided much helpful knowledge on the research history and pointed out some errors in the early version of this paper. I thank Dr. Sihon Crutcher for his kind help with improving the presentation of the paper.

\section{REFERENCES}

[1] J. M. Ball, Remarks on blow-up and nonexistence theorems for nonlinear evolution equations, Quart. J. Math., 28 (1977), 473-486. MR0473484 (57:13150)

[2] H. A. Levine, Instability and non-existence of global solutions to nonlinear wave equations of the form $P u_{t t}=-A u+F(u)$, Trans. Amer. Math. Soc., 192 (1974), 1-21. MR0344697 (49:9436)

[3] H. A. Levine, Some additional remarks on the nonexistence of global solutions to nonlinear wave equations, SIAM J. Math. Anal., 5 (1974), 138-146. MR.0399682 (53:3525)

[4] H. A. Levine, J. Serrin, Global nonexistence theorems for quasilinear evolution equations with dissipation, Arch. Rational Mech. Anal., 137 (1997), 341-361. MR1463799 (99b:34110)

[5] H. A. Levine, G. Todorova, Blow up of solutions of the Cauchy problem for a wave equation with nonlinear damping and source terms and positive initial energy, Proc. Amer. Math. Soc., 129 (2001), 793-805. MR1792187 (2001k:35212)

[6] V. Georgiev, G. Todorova, Existence of a solution of the wave equation with nonlinear damping and source terms, J. Differential Equations, 109 (1994), 295-308. MR.1273304 (95b:35141)

[7] R. T. Glassey, Blow-up theorems for nonlinear wave equations, Math. Z., 32 (1973), 183-203. MR0340799 (49:5549)

[8] F. Gazzola, M. Squassina, Global solutions and finite time blow up for damped semilinear wave equations, Ann. Inst. H. Poincaré Anal. Non Linéaire, 23 (2006), 185-207. MR2201151 (2007c:35118) 
[9] V. A. Galaktionov, S. I. Pohozaev, Blow-up and critical exponents for nonlinear hyperbolic equations, Nonlinear Analysis, 53 (2003), 453-466. MR.1964337 (2004b:35230)

[10] Jorge A. Esquivel-Avila, The dynamics of a nonlinear wave equation, Journal of Mathematical Analysis and Applications, 279 (2003), 135-150. MR1970496 (2004c:35278)

[11] L. E. Payne, D. H. Sattinger, Saddle points and instability of nonlinear hyperbolic equations, Israel. J. Math., 22 (1975), 273-303. MR0402291 (53:6112)

[12] L. E. Payne, D. H. Sattinger, Saddle points and instability of nonlinear hyperbolic equations, Israel J. Math., 22 (1975), 273-303. Reviewed by Howard A. Levine. MR 0402291 (53:6112)

[13] D. H. Sattinger, On global solution of nonlinear hyperbolic equations, Arch. Rat. Mech. Anal., 30 (1968), 148-172. MR0227616 (37:3200)

[14] B. Straughan, Further global nonexistence theorems for abstract nonlinear wave equations, Proc. AMS, 48 (1975), 381-390. MR0365265 (51:1518)

[15] M. Tsutsumi, On solutions of semilinear differential equations in a Hilbert space, Math. Japan, 17 (1972), 173-193. MR0355247(50:7723)

[16] G. Todorova, K. Christov, Existence and blow-up of solutions of some nonlinear parabolic problems, C. R. Acad. Bulgare Sci., 42 (1989), 17-20. MR.1027475 (91d:35109)

[17] G. Todorova, E. Vitillaro, Blow-up for nonlinear dissipative wave equations in $\mathbb{R}^{n}$, J. Math. Anal. Appl., 303 (2005), 242-257. MR2113879 (2005k:35292)

[18] Hiroshi Uesaka, Oscillation or nonoscillation property for semilinear wave equations, Journal of Computational and Applied Mathematics, 164-165 (2004), 723-730. MR2056910 (2005b:35195)

[19] E. Vitillaro, Global nonexistence theorems for a class of evolution equations with dissipation, Arch. Ration. Mech. Anal., 149 (1999), 155-182. MR1719145 (2000k:35205)

[20] Liu Yacheng, On potential wells and vacuum isolating of solutions for semilinear wave equations, J. Differential Equations, 192 (2003), 155-169. MR1987088 (2004h:35151)

[21] Liu Yacheng, Zhao Junsheng, On potential wells and applications to semilinear hyperbolic equations and parabolic equations, Nonlinear Analysis, 64 (2006), 2665-2687. MR.2218541 (2007a:35108)

[22] Liu Yacheng, Xu Runzhang, Wave equations and reaction-diffusion equations with several nonlinear source terms of different sign, Discrete and Continuous Dynamical System-Series B, 7 (2007), 171189. MR2257457 (2007h:35232) 